\title{
Gentamicin and Colistin in Chronic Purulent Bronchial Infections
}

\author{
A. PINES,* M.D., M.R.C.P.ED.; H. RAAFAT,* M.R.C.P.GLASG.; K. PLUCINSKI,* M.D.
}

Brit. med. F., 1967, 2, 543-545

Gentamicin is a recently discovered antibiotic produced from Micromonospora purpurea, highly active in vitro against such problem bacilli as Pseudomonas pyocyanea and Klebsiella pneumoniae (Klein et al., 1964 ; Barber and Waterworth, 1966). Infections with these organisms have been successfully treated with gentamicin given systemically, these involving the respiratory or urinary tracts (Klein et al., 1964 ; Brayton and Louria, 1964), or severe burns (Stone et al., 1965). Pseudomonads and klebsiellae are frequently isolated nowadays from the sputum of patients with chronic respiratory disorders; they often cause death (Pierce et al., 1966), and may be very difficult to eliminate (Pines et al., 1963). The in vitro and preliminary clinical experience described above suggested that gentamicin could be valuable in the treatment of these infections. We describe here our experience with this antibiotic. Colistin has been shown to be effective in certain Ps. pyocyanea infections (Halliday, 1967). We append a note on our experiences with colistin.

\section{Patients}

During the past 12 months we have admitted to our hospital a number of cases of persistent pseudomonas or klebsiella infections of the bronchi. The patients were ill, and had not improved despite intensive and varied treatment. The antibiotics unsuccessfully used had been penicillin (9-12 megaunits daily); streptomycin ( 1 g. daily); tetracycline, chloramphenicol, and sometimes erythromycin (2-3 g. daily); and ampicillin or cephaloridine (4-6 g. daily). All the patients had had at least four courses chosen from these antibiotics and some many more.

Thirty-six therapeutic trials of gentamicin, given by various routes, were made in 23 patients. All were men: nine were aged 50-59 years, ten aged $60-69$, and four 70 years or more. Seventeen weighed 50 to $70 \mathrm{~kg}$; the remaining six were below $50 \mathrm{~kg}$. Seven of the 23 had purulent bronchiectasis ; the remainder had chronic purulent bronchitis. Their general management included vigorous physiotherapy, bronchodilators, and digitalis and diuretics in some. Their illnesses were often very severe: four patients out of the eight originally selected for gentamicin treatment died while awaiting the first supplies of the drug.

\section{Methods of Assessment}

Where specific organisms were isolated they were regarded as significant only if they were in moderate or profuse growth in at least three pretreatment cultures. Three further specimens were examined after treatment. Standard methods of culture were used. Clinical assessment of the results of treatment was threefold and always blind, the assessor knowing nothing of each patient's treatment. (1) A 24-hour specimen of sputum was collected daily and examined independently by an experienced charge nurse for the degree of purulence and quantity. The presence or absence of pus was confirmed independently in the laboratory when examining the bacteriological specimens. (2) An independent assessment of the general state of the patient was made by one of us before and after treatment, and the

- Ware Park Hospital, Ware, Herts. patient's opinion of the treatment was also sought. (3) The maximal peak flow rate was estimated independently before and after treatment.

None of the patients had an eosinophilia in the sputum, and precipitin tests for aspergillin were negative in the six patients tested. Thus the only reason for the presence of pus in the sputum was bacterial infection. The blood urea in every case was normal before treatment. The serum transaminase (S.G.P.T. and S.G.O.T.) was estimated in 15 patients before and after treatment. In certain patients gentamicin was assayed in the blood and sputum by means of the "zone of inhibition well" technique with B. pumilus (N.C.T.C., 8241) (Weston, 1967).

\section{Treatment and Results}

Intramuscular Route.-The first 11 patients were given a moderately high dosage of gentamicin. One received $80 \mathrm{mg}$. three times daily, two $100 \mathrm{mg}$. twice daily, and eight $120 \mathrm{mg}$. twice daily for seven days, followed by the same or slightly lower amounts for another seven days. In three the sputum cleared and remained mucoid for one, three, and four weeks respectively; in eight there was no effect. Because of this failure we tried a higher dosage, $120 \mathrm{mg}$. three times daily for seven days, followed by the same or smaller quantities for another seven days. There were eight patients and in only one did the sputum become mucoid.

Aerosol Treatment.-Postulating that perhaps sufficient gentamicin was not penetrating through the bronchial barrier from the blood stream, we gave 11 patients aerosols of the antibiotic. The amount given was $20 \mathrm{mg}$. in $5 \mathrm{ml}$. of normal saline four times daily. In six this was given through positivepressure apparatus (the Bird or Emerson types); in five the Collison nebulizer was used. The course lasted for one to two months. In two the sputum became mucoid and remained so for two and five weeks respectively; in nine there was no response.

Combined Intramuscular and Aerosol Treatment.-Finally, $120 \mathrm{mg}$. of gentamicin was given twice daily intramuscularly along with an aerosol of the drug in the same dose as above, both for a fortnight. The sputum became mucoid in only one of the six patients treated.

\section{Clinical Results}

The bedside and laboratory assessments of the sputum for purulence were in agreement in each case. Changes in the quantity of the sputum were variable and inconstant. Among the seven patients whose sputum became mucoid the clinical assessor noted general improvement in six, and also in another seven patients whose sputum improved marginally only. All these patients stated that they felt better with treatment. We regard the complete disappearance of pus from the sputum as the most important and direct effect of the antibiotic and have taken this as the final basis of our assessment. No patients deteriorated.

The severity of the respiratory condition of these patients was shown by the maximal peak flow rate, which was below 
200 litres per minute in all. In those who achieved mucoid sputum the rate increased by 10 to $25 \%$ in four and was unchanged or had decreased in three. In the other patients there were no significant changes.

Thus among the 36 trials of treatment there was success in seven (19\%) and failure in 29 . The most remarkable success was in one patient who had extensive bilateral bronchiectasis and who had had purulent sputum for 30 years. After gentamicin intramuscularly the sputum became mucoid and remained so for four months afterwards ; this patient's general state was greatly improved.

\section{Clinical Results Related to Bacteriological Isolates and their Sensitivity to Gentamicin in 36 Treatments}

Sensitivity tests were done by means of a plate technique and discs impregnated with gentamicin at a concentration of 2 and $10 \mu \mathrm{g}$. per disc.

Ps. pyocyanea was isolated in 20 cases. In five the sputum became mucoid after treatment though in two cases of these the organisms could still be isolated in several specimens. Conversely, in three the organisms disappeared from the sputum after treatment though this remained purulent. None of the cultures were sensitive to the $2-\mu \mathrm{g}$. concentration, 16 were sensitive to $10 \mu \mathrm{g}$. (the five successes were among this group), and four were resistant to $10 \mu \mathrm{g}$.

$K l$. pneumoniae was found in seven cases: six of the cultures were sensitive to the $10-\mu \mathrm{g}$. concentration but treatment was unsuccessful in all six. One culture, not tested, was from the patient whose improvement is described above; no klebsiellae were isolated later.

Haemophilus influenzae was isolated in four cases, Proteus morganii in three, and Staphylococcus aureus in two, all sensitive to the $10-\mu \mathrm{g}$. concentration. There was improvement in one case of $H$. influenzae infection.

\section{Blood Levels and Toxicity}

Blood for gentamicin assay was taken one hour after injection, when the peak serum concentration is usually reached, and on the first and fourth days of treatment. With intramuscular therapy, blood levels of gentamicin were $10 \mu \mathrm{g}$. or more per ml. during treatment in six patients and in one rose to between 15.5 and $21 \mu \mathrm{g}$. Three of these received $120 \mathrm{mg}$. twice and three the same dose three times daily. In none was there evidence of renal disease. Dizziness accompanied by mild Rombergism occurred in three of these patients, and the S.G.O.T. rose in a fourth from 40 Reitman Frankel units before treatment to 46 units after a week, then 68 two weeks later, 72 a week after, and after a further two weeks had returned to 36 units. Lower levels of $7-9.5 \mu \mathrm{g} . / \mathrm{ml}$. were found in eight patients, three of whom were receiving high doses, and of $4-6.5 \mu \mathrm{g} . / \mathrm{ml}$. in seven patients, all on low doses: in none were there toxic effects. With inhalation therapy alone, blood levels were $2 \mu \mathrm{g} . / \mathrm{ml}$. in two patients and less than $0.2 \mu \mathrm{g} . / \mathrm{ml}$. in five others. With combined treatment the range of blood levels was the same as in those patients given intramuscular treatment alone.

Sputum levels one hour after aerosol treatment alone were $0.1-0.5 \mu \mathrm{g} . / \mathrm{ml}$.' in four patients, $1-2 \mu \mathrm{g} . / \mathrm{ml}$. in two patients, and $20 \mu \mathrm{g} . / \mathrm{ml}$. in one patient. Sputum levels after two hours were higher, usually $1-5 \mu \mathrm{g} . / \mathrm{ml}$., but in two patients were over $10 \mu \mathrm{g} . / \mathrm{ml}$. When intramuscular treatment alone was given, sputum levels in one patient were $0.1-0.5 \mu \mathrm{g} . / \mathrm{ml}$. and in another $1 \mu \mathrm{g} . / \mathrm{ml}$. ( $25 \%$ of the blood level) ; in two patients top levels of 5 and $6 \mu \mathrm{g} . / \mathrm{ml}$. were reached $(70-90 \%$ of the blood kevels). Only in the first patient was treatment successful.

Dizziness was noted in one patient receiving high doses, in whom blood levels had not been estimated. In all the patients with dizziness this disappeared within a few days after the end of treatment; caloric tests done at this time only confirmed this.

\section{Colistin}

Seventeen patients with purulent exacerbations of bronchitis were treated with colistin. Thirteen had persistent pseudomonas infections which had not responded to gentamicin. Four showed persistent and profuse sputum cultures of Escherichia coli ; infections with these organisms had sometimes responded to colistin (Pines et al., 1963). All the cultures were sensitive to discs containing $50 \mu \mathrm{g}$. of colistin. The patients' general features, their management, and their independent assessments were the same as in the gentamicin study. Four to six million units of colistin were given by the intramuscular route and two to four million by inhalation in a Collison or Bird apparatus, both daily in three or four divided doses for seven to ten days.

Treatment completely failed in all 13 patients with pseudomonas infections, the sputum and the clinical and patients' own assessments all agreeing. In one of the four patients with $E$. coli infection the sputum became mucoid for the first three only of the ten days of treatment ; there was no response in the other three patients. Five patients deteriorated seriously during the second to sixth days of treatment-three with peripheral circulatory collapse and fever. Inhalations were badly tolerated by three patients. No toxic effects appeared.

\section{Discussion}

Gentamicin failed to benefit most of our patients, despite high blood levels and adequate though variable penetration into the sputum. Various routes, such as intramuscular alone, aerosol alone, or both combined, were effective in occasional patients only. These results were rather worse than those recorded by others (Klein et al., 1964 ; Brayton and Louria, 1964), but it must be remembered that our patients had advanced disease and had not responded previously to the most intensive and varied antibiotic treatment.

The disc-sensitivity tests suggested that almost all of the bacteria isolated were relatively sensitive to gentamicin and might conceivably be inhibited by the concentrations reached in the blood. Why so few patients benefited is obscure. Binding of the antibiotic by mucus may have interfered with its action ; a mucolytic agent could conceivably help here, but we have not tried this. The antibiotic may have been prevented from reaching the organisms by fibrosis, relative avascularity, and the presence of bronchial abscesses (Reid, 1954), as we have postulated in similar advanced cases of bronchial infection (Pines et al., 1965).

The significance of Ps. pyocyanea and $\mathrm{Kl}$. pneumoniae in chronic purulent bronchial infections is still disputed. Their significance has sometimes been denied (May, 1953, 1954), but they are often found in profuse growth in these patients, particularly after antibiotic treatment (Pierce et al., 1966). Further, a characteristic necrotizing pneumonia due to these bacteria has been described at postmortem studies in such cases (Fraenkel, 1917 ; Pierce et al., 1966). Though gentamicin treatment is probably more effective than other specific antibiotics available, such as colistin, treatment of these infections in the bronchi still remains a problem.

If blood levels of $10 \mu \mathrm{g} . / \mathrm{ml}$. are not exceeded, the medium doses of gentamicin we have used appear safe, though in two patients on this dosage such levels were reached with resulting toxic effects. Higher levels of gentamicin resulted in more frequent toxicity and were no more effective. The doses of gentamicin we have used in these resistant infections have been several times those usually recommended. 
Colistin by combined intramuscular and aerosol treatment failed completely in all patients. The patients treated successfully by Halliday (1967) appear to have had acute illnesses which were not, as in ours, superimposed upon chronic infections. Our results confirm previous impressions (Pines et al., 1963) that colistin has little or no place in the treatment of purulent exacerbations of chronic bronchitis.

\section{Summary}

Thirty-six trials of gentamicin were made in 23 patients suffering from chronic purulent bronchial infections. Pseudomonas pyocyanea and Klebsiella pneumoniae had been repeatedly isolated in profuse growth. The routes were intramuscular alone in medium and high doses, inhalation alone, and both combined. In only seven (19\%) did the treatment succeed.

Transient toxic effects were noted in only four patients with blood levels of $10 \mu \mathrm{g}$. or more and in a fifth patient given high doses, where levels were not measured.

Colistin in high doses was given by intramuscular injection and inhalation together to 17 patients; none benefited.
Acknowledgements are due to Mr. J. H. Weston, L.Inst.Biol., Nicholas Research Institute Ltd., for blood and sputum levels recorded; to Dr. Ross Renton and Mr. G. A. Poulter for supplies of Gentacin and for generous help ; and to the medical, nursing, and secretarial staff of Ware Park Hospital and the laboratory staff of Hertford County Hospital for their assistance.

\section{REFERENCES}

Barber, M., and Waterworth, P. M. (1966), Brit. med. 7., 1, 203.

Brayton, R. G., and Louria, D. B. (1964). Arch. intern. Med., 114, 205. Fraenkel, E. (1917). Z. Hyg., 84, 369.

Halliday, N. P. (1967). Clin. Traals 7. In press

Klein, J. O., Eickhoff, T. C., and Finland, M. (1964). Amer. F. med. $S c l ., 248,528$.

May, J. R. (1953). Lancet, 2, 534.

- (1954). Ibid., 2, 839.

Pierce, A. K., Edmonson, E. B., McGee, G., Ketchersid, J., Loudon, R. G., and Sanford, J. P. (1966). Amer. Rev. resp. Dis., 94, 309.

Pines, A., Bundi, R. S., Greenfield, J. S. B., and Plucinski, K. (1965), Brit. F. Dis. Chest, 59, 81.

- Lydon, T. J., Plucinski, K., and Barkley, H. (1963). Practitioner, 190,502 .

Reid, L. M. (1954). Lancet, 1, 275.

Stone, H. H., Martin, J. D., Huger, W. E., and Kolb, L. (1965). Surg. Gynec. Obstet., 120, 351.

Weston, J. (1967). To be published.

\title{
Effect of Mental Stress on the Fibrinolytic Reactivity to Exercise
}

\author{
J. D. CASH, ${ }^{*}$ M.B., B.SC., M.R.C.P.ED. ; A. G. E. ALLAN,* F.I.M.L.T.
}

Brit. med.7., 1967, 2, 545-543

Nolf (1908) first proposed the existence of a dynamic equilibrium between fibrin deposition (coagulation) and removal (fibrinolysis). This hypothesis was extended by Astrup (1956), who envisaged a continuous deposition and removal of fibrin on the vessel walls as being a normal physiological process. $\mathrm{He}$ further argued that defective fibrinolysis might upset this "haemostatic balance," thus giving rise to excessive fibrin deposition, which could favour thrombosis and also be responsible for some forms of atherosclerosis according to the concept of Rokitansky (1852), elaborated by Duguid (1949, 1955). Subsequent efforts to demonstrate a defective fibrinolytic system in patients with occlusive vascular disease, by single sample analysis, have proved disappointing and controversial (Hume, 1958 ; Lackner and Merskey, 1960 ; Nestel, 1960 ; Ogston, 1962 ; Katz et al., 1963 ; Naimi et al., 1963 ; MacKay and Hume, 1964 ; Fearnley, 1965).

It is possible that some of the present uncertainty arises from the known fact that blood fibrinolytic activity may fluctuate, in any one person, from day to day and throughout the same day (Fearnley et al., 1957 ; Blix, 1961). Unless extreme and prolonged differences exist between patients with occlusive vascular disease and controls, comparison by single estimations could fail to provide satisfactory information. It is also possible that the significant difference between these two groups may not be found by a simple measurement of absolute resting values of fibrinolytic activity, but rather in the capacity of the fibrinolytic system to respond to physiological stimulants, including exercise (Biggs et al., 1947 ; Truelove, 1951 ; Fearnley and Lackner, 1955 ; Billimoria et al., 1959 ; Sherry et al., 1959 ; Ogston and Fullerton, 1961 ; Iatridis and Ferguson, 1963 ; Jang et al., 1964 ; Ogston and McAndrew, 1964 ; Burt et al.,

\footnotetext{
- From the South-east Scotland Regional Blood Transfusion Research
Laboratories, Royal Infirmary, Edinburgh.
}

1964) and mental anxiety (Macht, 1952; Dreyfuss, 1956 ; Friedman et al., 1958 ; Ogston et al., 1962).

Preliminary observations, made during the early part of a pilot study designed to test this hypothesis, showed that the fibrinolytic response (calculated as a percentage response of the pre-exercise level of the resting euglobulin lysis time) to a standardized submaximal exercise procedure was reproducible in any one individual; there were significant differences between individuals, and it was possible to isolate a group of poor responders (Cash, 1966). It was suggested that these poor responders had a defect in their mechanisms for the production of plasminogen activator to stress, and that they might therefore be more liable to episodes of an imbalance of the coagulationfibrinolysis equilibrium as envisaged by Astrup.

It was assumed that the ability to generate plasminogen activator after exercise was, in part, genetically determined. Subsequent studies, reported in this communication, would indicate that environmental factors, in the form of prolonged mental stress, may also be relevant.

\section{Subjects}

Five male medical student volunteers were studied before, during, and after a resit examination for the 2nd M.B., Ch.B Five male age-matched control volunteers were studied simultaneously-these were not sitting examinations but enjoying their summer vacation.

\section{Materials}

Anticoagulant.-Sodium citrate B.P. $3.8 \%$.

Buffers.- (a) Barbiturate buffer. Modified veronal buffer of pH 7.4 and ionic strength 0.15 (Owren, 1947). (b) Tris buffer. 